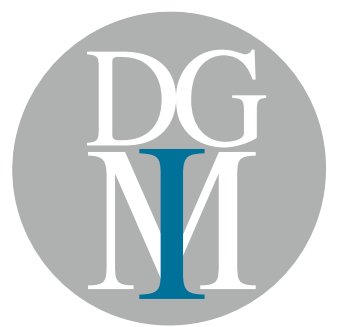

\title{
MITTEILUNGEN DER DEUTSCHEN GESELLSCHAFT FÜR INNERE MEDIZIN
}

\section{An die Mitglieder der DGIM}

T iebe Kolleginnen und Kollegen, _allen die zum Internistenkongress 2009 nach Wiesbaden gekommen sind, danke ich im Namen des Vorstandes der DGIM herzlich für ihr Interesse an unserer Jahrestagung.

Ebenso möchte ich mich im $\mathrm{Na}-$ men der Gesellschaft bei allen denjenigen von Ihnen entschuldigen, die wegen Überfüllung einzelner Räume keinen Platz fanden und entweder stehen mussten oder aber an ihrem ausgewählten Symposium nicht teilnehmen konnten. Glauben Sie mir - ich weiß, was es bedeutet: sich freizunehmen, den Dienst mit anderen aufzuteilen, die Fahrt- und Hotelkosten zu tragen, für sich nach dem Programm eine eigene Auswahl von Symposien zu treffen - und dann keinen Platz zu finden. Sie mögen mir ebenfalls glauben, das dies dem Generalsekretär einer Gesellschaft wehtut. Präsident und Kongressteam haben sich große Mühe gegeben, die Vortragssäle den Themen entsprechend nach Möglichkeiten der Kongresshalle auszuwählen. Dies wird auch für den Internistenkongress 2010 so sein. Dennoch - ein gewisses Problem wird bis zu der von der Stadt Wiesbaden geplanten Neugestaltung der Rhein-Main-Hallen bestehen bleiben, bedingt durch das vorgegebene Hallendesign. Wir werden gemeinsam mit dem Kongressveranstalter alles daran setzen, mit dem Problem fertig zu werden.

Bleiben Sie dem Internistenkongress treu!

Prof. Dr. Hans-Peter Schuster, Hildesheim, Generalsekretär der DGIM
Cehr geehrte Damen und Herren vom Kongressteam, und Vorstand der DGIM

es ist schon über 1 Woche her, dass meine Frau, unser Sohn und ich aus Wiesbaden zurückgekehrt sind. Unser Sohn $\left(2 \frac{1}{2}\right.$ Jahre alt) erzählt immer noch von, Wiesbaden“. Dass der jährliche
Kongress immer ein Highlight ist, werden Sie sicher von vielen Kollegen gehört haben.

Wir möchten Ihnen ganz besonders für die tolle Kinderbetreuung danken.

Unser Sohn war an allen 5 Kongresstagen sehr gut betreut, hatte viel
Spaß und konnte sich abends kaum trennen. Ohne dieses tolle (und obendrein auch noch kostenlose) Angebot hätte nur einer von uns am Kongress teilnehmen können.

Mit freundlichen Grüßen aus Aalen, Familie Meiborg 


\section{Neuwahlen in die Gremien der DGIM}

A nlässlich der Sitzungen von Vorstand und Ausschuss und der Ordentlichen Mitgliederversammlung der Deutschen I Gesellschaft für Innere Medizin während des 115. Jahreskongresses in Wiesbaden 2009 fanden Wahlen zu Gremien der DGIM statt. Im Folgenden informieren wir über die neue Zusammensetzung der betroffenen Gremien.

\section{Vorstand}

Satzungsgemäß ist der bisherige 1. stellvertretende Vorsitzende Prof. Dr. G. Ertl (Würzburg) aus dem Vorstand ausgeschieden. Neu in den Vorstand gewählt wurde Prof. Dr. J. Mössner (Leipzig).

Dem neuen Vorstand 2009/2010 gehören somit an:

Prof. Dr. J. Schölmerich (Regensburg) als Vorsitzender

Prof. Dr. R.E. Kolloch (Bielefeld) als 1. stellvertretender Vorsitzender Prof. Dr. H. Lehnert (Lübeck) als 2. stellvertretender Vorsitzender Prof. Dr. J. Mössner (Leipzig) als 3. stellvertretender Vorsitzender Prof. Dr. H.-P. Schuster (Hildesheim) als Generalsekretär Prof. Dr. R. Raedsch (Wiesbaden) als Kassenführer

\section{Geschäftsführer}

RA M.G. Broglie (Wiesbaden)

\section{Ausschuss}

Satzungsgemäß sind aus dem Ausschuss ausgeschieden: Prof. Dr. G. Höffken (Dresden), Prof. H.-R. Figulla (Jena), Prof. Dr. U. Frei (Berlin), Prof. Dr. H. Zeidler (Hannover). Als neue Ausschussmitglieder wurden gewählt: Prof. Dr. M. Stumvoll (Leipzig), Prof. Dr. G. Hasenfuß (Göttingen), Prof. Dr. J.F.E. Mann (München), Prof. Dr. U. Müller-Ladner (Bad Nauheim), Prof. Dr. M. Borggrefe (Mannheim).

Weiterhin gehören dem Ausschuss an: Prof. Dr. E. Pilger (Graz) für die Österreichische Gesellschaft für Innere Medizin (ÖGIM) und Prof. Dr. E. Battegay (Zürich) für die Schweizerische Gesellschaft für Innere Medizin (SGIM).

Der Ausschuss hat somit folgende von der Gesellschaft gewählte Mitglieder

Prof. Dr. E. Battegay (Zürich)

Prof. Dr. M. Borggrefe (Mannheim)
Prof. Dr. J. Floege (Aachen)

Prof. Dr. M. Hallek (Köln)

Prof. Dr. G. Hasenfuß (Göttingen)

Prof. Dr. F. Herth

(Heidelberg-Rohrbach)

Porf. Dr. G. Hintze (Bad Oldesloe)

Prof. Dr. J.F.E. Mann (München)

Prof. Dr. E. Märker-Hermann

(Wiesbaden)

Prof. Dr. U. Müller-Ladner

(Bad Nauheim)

Prof. Dr. L. Pientka (Bochum)

Prof. Dr. E. Pilger (Graz)

Prof. Dr. P.-M. Schumm-Dräger

(München-Bogenhausen)

Prof. Dr. M. Stumvoll (Leipzig)

Prof. Dr. N. Suttorp (Berlin)

Prof. Dr. W. Vogel (Innsbruck)

Prof. Dr. B. Wörmann

(Braunschweig)

Prof. Dr. A.M. Zeiher

(Frankfurt am Main)

Dem Ausschuss gehören als die Repräsentanten der Schwerpunkte der Inneren Medizin an

Prof. Dr. A. Creutzig (Hannover), Angiologie

Prof. Dr. K. Mann (Essen),

Endokrinologie

Prof. Dr. W. Schepp

(München-Bogenhausen),

Gastroenterologie und Hepatologie

Prof. Dr. G. Ehninger (Dresden),

Hämatologie und Onkologie

Prof. Dr. M. Kelm (Aachen),

Kardiologie

Prof. Dr. H. Pavenstädt (Münster),

Nephrologie

Prof. Dr. M Pfeifer (Donaustauf),

Pneumologie

Prof. Dr. W.L. Gross (Lübeck),

Rheumatologie

Prof. Dr. W.V. Kern (Freiburg),

Infektiologie

Prof. Dr. H.-J. Trappe (Herne),

Internistische Intensivmedizin

Prof. Dr. C. Sieber

(Nürnberg),

Geriatrie
Dem Ausschuss gehören als

Repräsentant des Berufsverbandes

Deutscher Internisten (BDI) an

Dr. W. Wesiack (Hamburg)

und als Repräsentant der

Korporativen Mitglieder

Dr. F.-J. Wingen (Leverkusen), Bayer Vital GmbH

Komitee Theodor-Frerichs-Preis

In das Frerichs-Preis-Komitee 2009/2010 wurden gewählt: Prof. Dr. M. Hallek (Köln), Vorsitzender

Prof. Dr. M. Reincke (München)

Prof. Dr. H. Pavenstädt (Münster)

Prof. Dr. M. Zeitz (Berlin)

Prof. Dr. G. Nickenig (Bonn)

Komitee

Präventionspreis Innere Medizin

Für das Präventionspreis-Komitee 2009/2010 wurden bestätigt:

Prof. Dr. Dr. h.c. mult. H.G. Lasch (Gießen)

Prof. Dr. D. Klaus (Dortmund)

Prof. Dr. H. Greten (Hamburg)

Prof. Dr. U.R. Fölsch (Kiel)

Prof. Dr. M. Weber (Köln)

Kommission

„Struktur - Krankenversorgung“

Prof. Dr. M.P. Manns (Hannover), Vorsitzender

Prof. Dr. P.-M. Schumm-Dräger

(München-Bogenhausen), stellvertretende Vorsitzende

Prof. Dr. N. Suttorp (Berlin)

Prof. Dr. M. Weber (Köln)

Prof. Dr. B. Wiedenmann (Berlin)

Prof. Dr. G. Hasenfuß (Göttingen)

Prof. Dr. M. Freund (Rostock)

Dr. K. Reinhardt (Bielefeld)

Prof. Dr. K. Mann (Essen) 


\section{Kommission}

"Wissenschaft - Nachwuchsförderung"

Prof. Dr. Elisabeth Märker-Hermann (Wiesbaden), Vorsitzende

Dr. A. Althoff (Gießen)

PD Dr. J. Hampe (Kiel)

Prof. Dr. R. Knöll (Göttingen)

Prof. Dr. F. Lammert

(Homburg/Saar)

Prof. Dr. G. Nickenig (Bonn)

Prof. Dr. N. Schmitz (Hamburg)

Prof. Dr. E. Schulze-Lohoff

(Duisburg)

Prof. Dr. E. Stüber (Warendorf)

Prof. Dr. M. Schott (Düsseldorf)

PD C. Espinola-Klein (Mainz)

Prof. Dr. G. Fätkenheuer (Köln)

PD Dr. J. Graf (Marburg)

\section{Kommission}

„Diagnostik - Therapie“

Prof. Dr. J. Mössner (Leipzig)

(Leitlinienbeauftragter), Vorsitzender

Prof. Dr. G. Ehninger (Dresden)

Prof. Dr. H. Klein (Bochum)

Prof. Dr. M. Borggrefe (Mannheim)

Prof. Dr. M. Reincke (München)

Herausgebergremium

„Rationelle Diagnostik und Therapie

in der Inneren Medizin“"

Prof. Dr. J. Meyer (Mainz),

federführend

Prof. Dr. R. Dierkesmann (Stuttgart)

Prof. Dr. W.E. Fleig (Leipzig)

Prof. Dr. H. Heidrich (Berlin)

Prof. Dr. H. Heimpel (Ulm)

Prof. Dr. W. Kern (Freiburg)

Prof. Dr. O.A. Müller (München)

PD Dr. Ch. Specker (Essen)

Prof. Dr. Chr. Wanner (Würzburg)

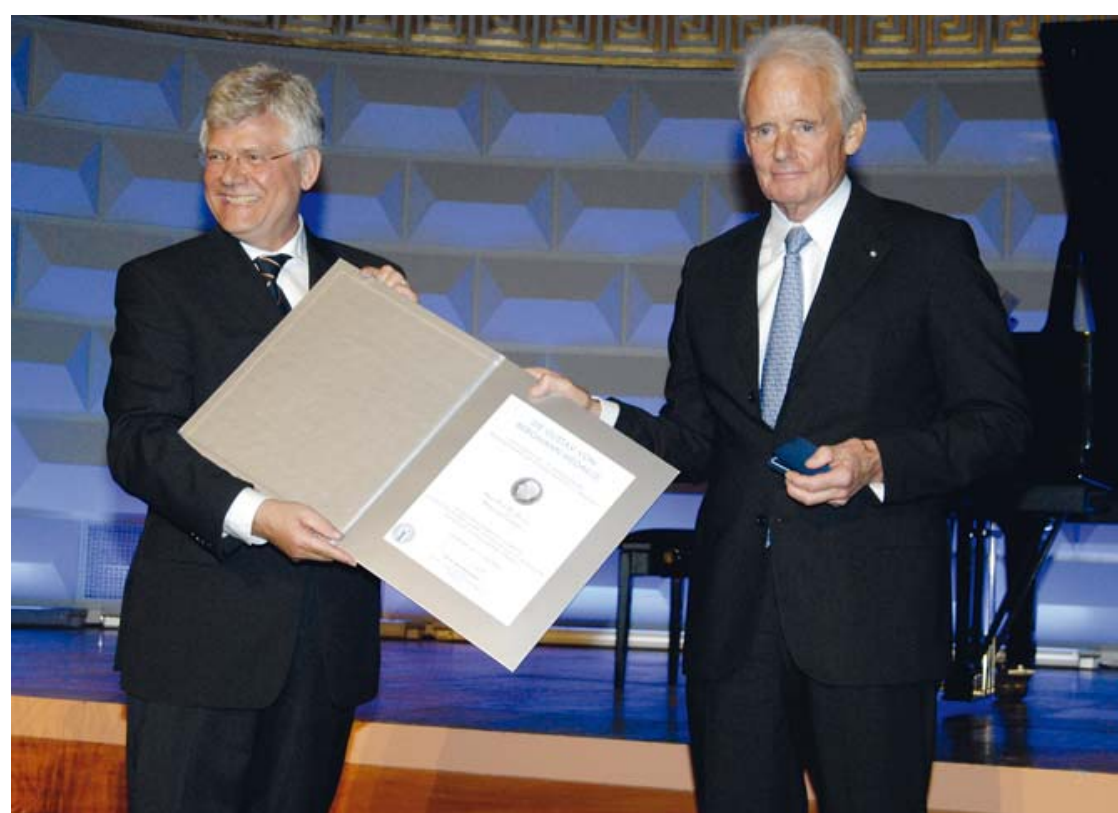

Professor R.E. Kolloch (links), Bielefeld, bei der Verleihung der Gustav-von-BergmannMedaille an Professor M. Classen, München.

Leitlinienbeauftragter

Prof. Dr. J. Mössner (Leipzig)

Beauftragter Korporative Mitglieder

Prof. Dr. U.R. Fölsch (Kiel)

\section{Stiftungsbeauftragter}

Deutsche Stiftung Innere Medizin, Prof. Dr. M. Weber (Köln)

Träger der

Gustav-von-Bergmann-Medaille

2009 Meinhard Classen (München) 2008 Peter C. Scriba (München)
2007 Volker Diehl (Berlin) Hans-Peter Schuster (Hildesheim)

2005 Karl-Hermann Meyer zum Büschenfelde (Berlin)

2004 Werner Creutzfeldt† (Göttingen)

2001 Hanns Gotthard Lasch (Gießen) Wolfgang Gerok (Freiburg)

2000 Paul Schölmerich (Mainz) Walter Siegenthaler (Zürich)

1999 Rudolf Gross (Köln)

1996 Gotthard Schettler (Heidelberg) 1994 Hans Erhard Bock †(Tübingen) Eine vollständige Auflistung aller Gremien der DGIM finden Sie in der Jahresbroschüre 2009

Prof. Dr. H.-P. Schuster, Hildesheim, Generalsekretär der DGIM 


\section{Theodor-Frerichs-Preis 2009}

$\mathrm{D}$ ie Deutsche Gesellschaft für Innere Medizin e.V. (DGIM) verleiht jährlich den mit 20000 Euro dotierten Theodor-Frerichs-Preis für die beste vorgelegte, möglichst klinischexperimentelle Arbeit auf dem Gebiet der Inneren Medizin. Die diesjährige Preisverleihung fand während des 115 . Kongresses für Innere Medizin in Wiesbaden statt. Auf Vorschlag der Jury prämierte die Deutsche Gesellschaft für Innere Medizin

PD Dr. med. Marco Idzko Universitätsklinikum Freiburg, Abteilung für Pneumologie für die Arbeit

Extracellular ATP triggers and maintains asthmatic airway inflammation by activating dendritic cells.

Die von Herrn PD Dr. Marco Idzko vorgelegte Arbeit befasst sich mit der klinischen Relevanz von im biologischen System ubiquitär vorkommenden Nukleotiden, wie z.B. ATP (Adenosin-5'-triphosphat). Insbesonde-

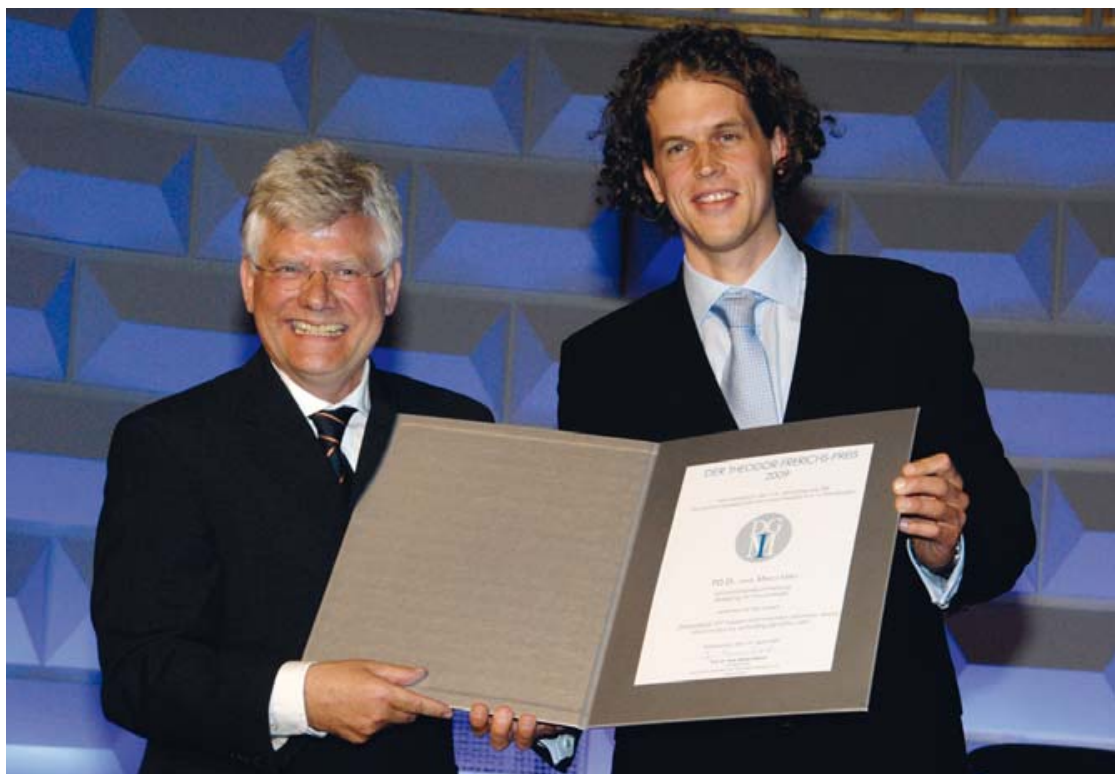

Professor R.E. Kolloch (links), Bielefeld, bei der Verleihung des Theodor-Frerichs-Preises an PD Dr. M. Idzko, Freiburg. re in chronisch entzündeten humanen Geweben liegen diese Nukleotide extrazellulär in hohen Konzentrationen vor, so dass sie ihrerseits die Entzündung über die Bindung an purinerge P2-Rezeptoren aktiv unterhalten können. Die Arbeitsgruppe von Herrn Idzko konnte bereits in Vorarbeiten zeigen, dass sämtliche strukturellen und inflammatorischen Zellen, die an der Pathogenese der asthmatischen Entzündungsreaktion beteiligt sind, funktionelle P2-Rezeptoren exprimieren und dass über deren Aktivierung in vitro eine ,,proasthmatische" Immunantwort ausgelöst werden kann. In der vorliegenden Arbeit zeigen die Autoren nun, dass die Allergenprovokation beim Menschen und im experimentellen Asthma-Mausmodell zu einer Freisetzung von endogenem ATP führt und dass hierbei die intrapulmonalen ATP-Level mit der Eosinophilie in der bronchoalveolären Lavage korrelieren. Gleichzeitig wiesen die Autoren nach, dass eine Reduktion der endogenen pulmonalen ATP-Spiegel sowie eine spezifische Inhibition der
P2-Rezeptoren während der Allergenprovokation zu einer Hemmung der asthmatischen Entzündungsreaktion beitragen. Mechanistisch identifizierten Idzko und Mitarbeiter myeloide dendritische Zellen als mögliche Zielzellen für das endogen produzierte ATP, die bekannterweise eine essentielle Rolle bei der Induktion und Unterhaltung der asthmatischen Entzündungsreaktion spielen.

Zusammenfassend zeigen die von Herrn PD Dr. Idzko publizierten Ergebnisse erstmals, dass die Allergenprovokation zu einer Freisetzung von endogenem ATP führt, welches selbst wiederum durch die Aktivierung von dendritischen Zellen die asthmatischen Entzündungsreaktionen induziert und unterhält. Die Autoren schlussfolgern aus ihren Untersuchungen, dass P2Rezeptor-Antagonisten somit eine neue Therapieoption für das steroidrefraktäre Asthma bronchiale darstellen.

Die von Herrn PD Dr. Idzko publizierten Ergebnisse sind hochinnovativ und ein Paradebeispiel für eine exzellente translationale Forschung von der Zellkultur, über das Mausmodell bis hin zum Patienten mit dem Ziel, aus pathophysiologischen Vorgängen eine therapeutische Intervention abzuleiten. Hervorzuheben ist, dass Herr PD Dr. Idzko bereits in den Jahren 2006 und 2007 zwei hochkarätige Publikationen im Journal of Clinical Investigation publiziert hat und derzeit durch ein EmmyNöther-Stipendium der Deutschen Forschungsgemeinschaft unterstützt wird. Mit 32 Jahren ist er gleichzeitig der jüngste Bewerber für den diesjährigen Theodor-Frerichs-Preis. Zusammenfassend ist somit nicht nur die Arbeit selbst die hochkarätigste unter den eingegangenen Bewerbungen, sondern auch der wissenschaftliche Werdegang und die bisherigen Forschungsleistungen von Herrn PD Dr. Idzko, auch unter Berücksichtigung seines Alters, unter den übrigen Bewerberinnen/Bewerbern hervorstechend und herausragend. 


\section{Präventionspreis Innere Medizin 2009}

Di er von der Deutschen Stiftung Innere Medizin über die Deutsche Gesellschaft für Innere Medizin verliehene und mit 5000 Euro dotierte Präventionspreis Innere Medizin wird für die beste aus dem deutschsprachigen Raum vorgelegte Arbeit auf dem Gebiet der Prävention innerer Krankheiten verliehen. Die diesjährige Preisverleihung fand anlässlich des 115 . Kongresses für Innere Medizin in Wiesbaden statt.

Preisträger ist

PD Dr. med.

Jochen Müller-Ehmsen

Klinikum der Universität Köln, Klinik III für Innere Medizin für die Arbeit

Decreased number of circulating progenitor cells in obesity: beneficial effects of weight reduction.

Die Arbeit untersucht zirkulierende Progenitorzellen bei Übergewicht und kommt zu wichtigen neuen, auch international bereits publizierten Ergebnissen, die durchaus den Charakter einer Präventionsarbeit haben. Die Anzahl zirkulierender endothelialer Progenitorzellen im peripheren Blut ist ein zellulärer Marker für das individuelle kardiovaskuläre Risiko. Je geringer die Zahl, desto höher das Risiko. In der vorliegenden Studie wurde geprüft, ob die Zahl der zirkulierenden Progenitorzellen bei Übergewichtigen, einem Kollektiv mit erhöhtem kardiovaskulären Risiko, vermindert ist oder ob dieser Effekt eine Bedeutung bei der Entstehung der Arteriosklerose hat und ob er durch Gewichtsverlust schon reversibel ist.

Dazu wurde im peripheren Blut von 149 freiwilligen Teilnehmern eines Diätprogramms die Zahl der CD34-, KDR/CD34-, CD133/CD34- und CD117/C34-positiven Zellen mittels Durchflusszytometrie bestimmt. Die Zahl der Progenitorzellen wurde zusätzlich als Zahl an fibronektinadhärenten Zellen und als Zahl der gebildeten solitären Kolonien in der Zellkultur notiert.

Tatsächlich wurden bei den Probanden sonographisch die Intima-Media-Dicke und die Endothelfunktion (,flow-mediated dilation“) als frühe
Arteriosklerosezeichen bestimmt. Nach einem 6-monatigen Diät- und Sportprogramm wurden die gleichen Parameter bei 86 Probanden mit einem mittleren Gewichtsverlust von 5,8 \pm $0,6 \mathrm{~kg}$ erhoben.

Es zeigte sich eine negative Korrelation aller untersuchten Zellpopulationen mit dem BMI und dem Hüftumfang. Ebenso waren die Anteile der betrachteten Subpopulationen an den CD34-positiven Zellen bei hohem Körpergewicht signifikant vermindert. Verschiedene Zellpopulationen reagierten dabei unterschiedlich stark auf das Vorliegen von Übergewicht. Bei den KDR/CD34-positiven Zellen zeigte sich eine BMI-abhängige Reduktion besonders deutlich.

Darüber hinaus bestanden eine positive Korrelation zwischen mittlerem Bauchumfang und Intima-Media-Dicke, zwischen Bauchumfang und Endothelfunktion und eine umgekehrte/ negative Korrelation zwischen IntimaMedia-Dicke in der Zahl der CD34und der CD117/CD34-positiven Zellen. Nach der Diät fand sich eine vom Ausmaß der Gewichtsreduktion sehr abhängige Zunahme der Progenitorzellen. Auch die mittlere Anzahl der CD133/CD34-positiven Zellen im Ge-

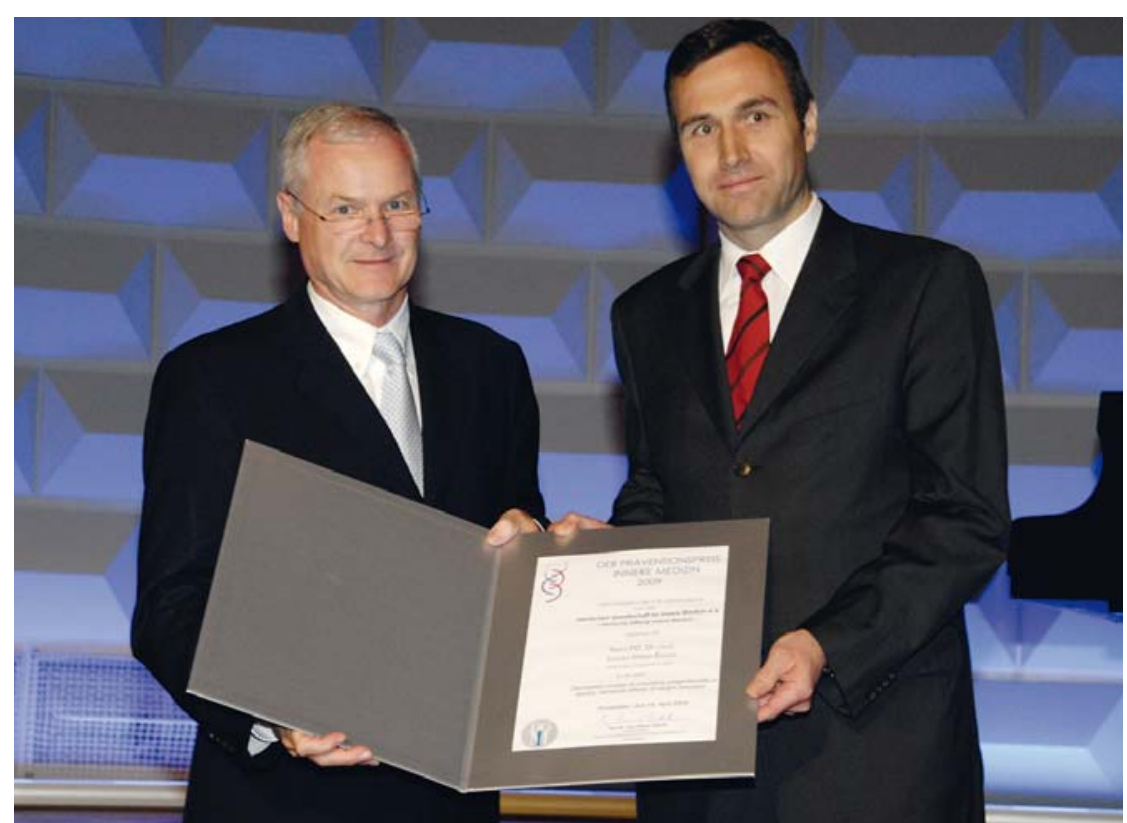

Professor M. Weber (links), Köln, bei der Verleihung des Präventionspreises Innere Medizin an PD J. Müller-Ehmsen, Köln. samtkollektiv war nach der Diät signifikant erhöht, wohingegen die KDR/ CD34-positiven Zellen unverändert blieben.

Interessanterweise zeigte sich dieser Zusammenhang nicht nur bei den Probanden, die ihre sportliche Aktivität gesteigert hatten, sondern auch bei den Probanden ohne gesteigerte sportliche Aktivität.

Diese Ergebnisse zeigen erstmals, dass Übergewicht (ähnlich anderen kardiovaskulären Risikofaktoren) mit einer reduzierten Anzahl zirkulierender Progenitorzellen assoziiert ist. Nach einer erfolgreichen Diät sind die Veränderungen $\mathrm{zu}$ einem Teil reversibel. Danach könnte der präventive Nutzen einer Gewichtsreduktion (ebenso wie der einer sportlichen Aktivität) durch eine erhöhte Zahl zirkulierender endothelialer Progenitorzellen entstehen. Dieser Marker hat durchaus präventiven Charakter und ist unter die bekannten Vorsorgefaktoren einzureihen.

Die Arbeit zeichnet sich durch eine saubere Methodik, eine gute statistische Auswertung und eine kritische Stellungnahme aus und führt $\mathrm{zu}$ innovativen, klinisch sehr wichtigen Ergebnissen. 


\section{Young Investigators' Award 2009}

$\mathrm{D}$ e jeweils besten Abstracts von Autoren unter 35 Jahren aus den jeweiligen Schwerpunkten nehmen am Young Investigators' Award teil (Angiologie, Intensiv- und Notfallmedizin, Pneumologie, Infektiologie, Gastroenterologie, Rheumatologie, Nephrologie, Kardiologie, Hämatoonkologie, Endokrinologie). Die Jury hat für den Young Investigators' Award 2009 folgende Preisarbeiten ausgewählt:

\section{Preis}

\section{Angiologie}

Dipl.-Biol. Wiebke Bielenberg miRNAs regulieren die Funktion glatter Gefäßmuskelzellen währen der Atherosklerose und Restenose

W. Bielenberg ${ }^{1}$, A. Prock ${ }^{1}$, H. Tillmanns $^{1}$, D. Sedding ${ }^{1}$

${ }^{1}$ Kardiologie/Angiologie, Universitätsklinikum Gießen, Innere Medizin I, Gießen

Introduction: Micro RNAs (miRNAs) are implicated to regulate mRNA levels of up to $30 \%$ of mammalian genes, comprising key regulators for cellular function. Maturation and expression of miRNAs is tightly controlled by the two processing enzymes Dicer and Drosha. Up to now, the role of miRNAs for vascular smooth muscle cell (VSMC) function remains elusive. Thus, the aim of this study was to evaluate the regulation and impact of Dicer and Drosha for smooth muscle cell function during the development of neointimal lesions in vitro and in vivo.

Methods and Results: Following wireinduced injury of the femoral artery of C57BL/6N mice, Dicer or Drosha mRNA expression was analyzed by realtime PCR in the developing neointimal lesions. Expression of Dicer and Drosha was significantly downregulated at day 10 and 21 following injury. Microarray analysis of miRNA expression at these time points revealed a significant downregulation of miRNAs implicated to regulate key molecules of cell cycle progression. Indeed, siRNA-mediated knockdown of Dicer or Drosha in human coronary SMC significantly augmented the growth factor-induced expression of CDK4, CDK6, SKP2, accelerated cell cycle progression as deter-

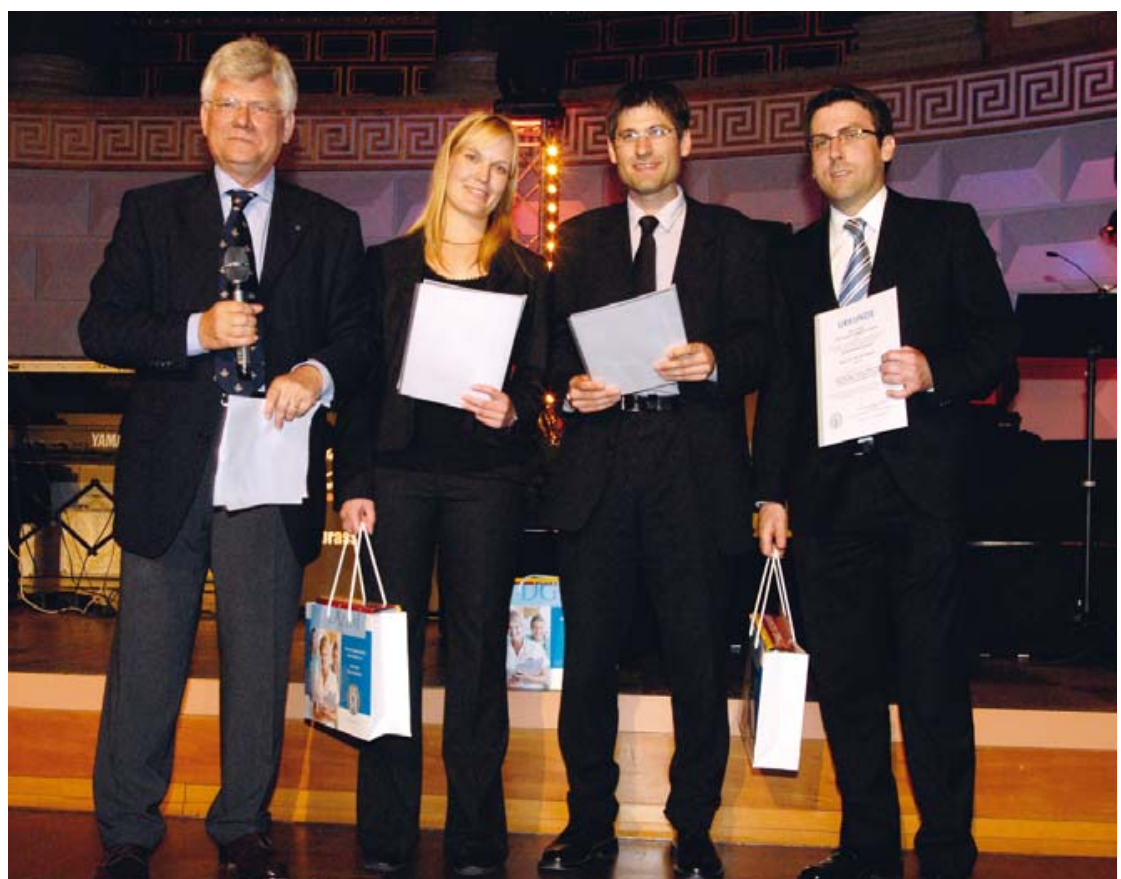

Von links Professor R.E. Kolloch, Dipl.-Biol. W. Bielenberg, Dr. A. Aghdassi, Dr. K. Hildner. mined by FACS analysis and increased VSMC proliferation as determined by total cell count as well as BrdU incorporation (Drosha: $0.0983 \pm 0.0194$ vs. $0.0527 \pm 0.0115$; Dicer: $0.0945 \pm 0.0172$ vs. $0.0527 \pm 0.0115 ; \mathrm{p}<0.005)$. Interestingly, knock down of Dicer and Drosha had no effect on migration or apoptosis. Among different stimuli, growth factors were identified as potent regulators of Drosha and Dicer expression.

In vivo, a knock down of Dicer and Drosha after wire injury led to an accelarated neointima formation.

Conclusions: These data indicate that the key miRNA-processing enzymes Dicer and Drosha are downregulated in neointimal lesions, most likely by mitogenic stimuli. Moreover, altered expression of these enzymes and of miRNAs seems to regulate VSMC function during the development of vascular proliferative diseases.

\section{Preis}

\section{Gastroenterologie}

Dr. med. Ali Aghdassi

Histon-Deacetylasen regulieren die ECadherin-Genexpression und erhöhen die Tumorigenität des Pankreaskarzinoms

A. Aghdassi ${ }^{1}$, U. Weiss ${ }^{1}$, C.-O. Behn ${ }^{1}$, J. Mayerle ${ }^{1}$, M.W. Büchler ${ }^{2}$, H. Friess ${ }^{3}$, C.-D. Heidecke ${ }^{4}$, M.M. Lerch ${ }^{1}$

${ }^{1}$ Klinik und Poliklinik für Innere Medizin A, Greifswald;

${ }^{2}$ Chirurgische Klinik, Universitätsklinikum Heidelberg, Heidelberg;

${ }^{3}$ Chirurgische Klinik und Poliklinik, Klinikum rechts der Isar, München; ${ }^{4}$ Abteilung für Allgemeine Chirurgie, Viszeral-, Thorax- und Gefäßchirurgie, Klinik und Poliklinik für Chirurgie, Greifswald

Einleitung: Das Pankreaskarzinom zählt zu den häufigsten gastroenterologischen Tumoren und zeichnet sich nach wie vor durch eine hohe Therapieresistenz aus. Ein schnelles Tumorwachstum und eine frühzeitige Metastasierung tragen besonders zur Aggressivität bei. Hierfür verantwortlich ist unter anderem ein Verlust von ZellZell-Kontakten zwischen Tumorzellen, 
insbesondere von E-Cadherin (CDH1), dem als transmembranären Protein eine besondere Bedeutung beim Zusammenhalt von Tumorzellverbänden zukommt. Warum manche Tumoren kein E-Cadherin exprimieren und welche Mechanismen dafür verantwortlich sind, ist kaum untersucht.

Ziel und Methoden: Ziel dieser Studie ist die Identifizierung von Regulationsmechanismen der E-Cadherin-Expression im Pankreaskarzinom. Hierzu wurden sowohl humane Pankreaskarzinomzellinien als auch Tumorgewebeproben untersucht, die einen $\mathrm{Tu}-$ morgehalt von über $50 \%$ aufwiesen.

Ergebnisse: Ca. 40\% der Tumorproben wiesen keine E-Cadherin-Expression auf. Die Sequenzierung aller Exons sowie eine methylierungsspezifische PCR nach DNA-Bisulfit-Konversion zeigte, dass weder somatische Mutationen noch Promotor-Hypermethylierung einen wesentlichen Einfluss auf die Regulation von E-Cadherin haben. Ebenso wenig waren in Gewebeproben Unterschiede des E-Cadherin-reprimierenden Transkriptionsfaktors SNAIL nachweisbar. Fluormetrische Messungen zeigten, dass im Pankreaskarzinom Histon-Deacetylasen hochreguliert sind. Außerdem führte die Inkubation von E-Cadherin-defizienten pankreatischen Karzinomzelllinien mit verschiedenen Histon-Deacetylase-Inhibitoren (HDACi) zu einer Reexpression von E-Cadherin. Eine Bindung acetylierter Histone an den E-Cadherin-Promotor führte zur Aktivierung, die Bindung der HistonDeacetylasen 1 und 2 zu einer Verminderung der Genexpression, wie wir durch Chromatin-Immunpräzipitation (ChIP) zeigen konnten. Zudem führten HDACi zu einer Verminderung der Zellproliferation und -migration in Pankreaskarzinomzelllinien.

Zusammenfassung: Diese Beobachtungen legen nahe, dass im Pankreaskarzinom E-Cadherin weniger durch somatische, sondern vielmehr durch epigenetische Mechanismen kontrolliert wird. Da diese Prozesse reversibel sind, liegt in der Verwendung von HDACi ein besonderes therapeutisches Potential für die Behandlung des Pankreaskarzinoms.

\section{Preis}

Hämatoonkologie

Dr. med. Kai Hildner

Batf 3 deficiency reveals a critical role for CD8a+ dendritic cell subsets in priming cytotoxic $T$ cell responses to tumors

K. Hildner ${ }^{1}$, B.T. Edelson ${ }^{2}$, W.E. Purtha $^{3}$, M. Diamond ${ }^{2}$, H. Matsushita ${ }^{2}$, M. Kohyama ${ }^{4}$ B. Calderon ${ }^{2}$, B. Schraml $^{2}$, E.R. Unanue ${ }^{2}$, M.S. Diamond $^{5}$, R.D. Schreiber ${ }^{2}$, T.L. Murphy $^{2}$, K.M. Murphy ${ }^{6}$

${ }^{1}$ Department of Pathology and Immunology and Howard Hughes Medical Institute and I. Med. Clinic, Johannes Gutenberg University, St. Louis, MO, USA, Mainz;

${ }^{2}$ Department of Pathology and Immunology, St. Louis, MO, USA;

${ }^{3}$ Departments of Medicine, St. Louis, MO, USA;

${ }^{4}$ Department of Pathology and Immunology and Howard Hughes Medical Institut, St. Louis, MO, USA;

${ }^{5}$ Departments of Medicine, St. Louis, MO, USA;

${ }^{6}$ Department of Pathology and Immunology and Howard Hughes Medical Institute, St. Louis, MO, USA

Zielsetzung und Methoden: Crosspresentation of exogenous antigens by MHC class I molecules to CD8+ T cell is a well established pathway, but its in vivo role in immunity to pathogens and tumors is less clear. Dendritic cells (DCs) contain heterogeneous subsets suggested to play distinct roles in immune responses, including initiation of T cell immunity. The CD8a+ DC subset carries out cross-presentation more efficiently than other subsets, but evi- dence for this activity has so far been based on in vitro or ex vivo observations, and has not been directly examined in vivo or in the context of tumor immunity. Demonstration of non-redundant activities of DC subsets is hampered by lack of systems allowing their elimination in vivo.

Global gene expression profiling across a panel of highly purified immune cells and non-immune tissues was performed to identify novel transcription factors critical for immune cell lineage commitment. A novel member of the AP-1 family of transcription factors, BATF3, with a unique, however distinct expression profile among immune cell subsets was identified and analyzed by generating gene knockout mice.

Ergebnis: Here, we report that mice deficient in the transcription factor Batf3 (p21SNFT) exhibit a lineage ablation of CD8a+ DCs but with sparing of the major CD4+ cDC and plasmacytoid dendritic cells (pDCs), and without other immune or somatic defects. Batf3-/- mice have APCs defective in cross-presentation and selectively lack virus-specific CD8+ T cell responses, indicating a requirement for $\mathrm{CD} 8 \mathrm{a}+\mathrm{DC}$ in these activities in vivo. Importantly, rejection of highly immunogenic syngeneic tumors was completely lacking in Batf3-/- mice, indicating a non-redundant role for crosspresenting CD8a+ DC for tumor rejection as well.

Schlussfolgerung: Batf3-/- mice may provide a useful system in which to evaluate the requirement for CD8a+ DC and cross-presentation to various pathogens and vaccines.

This work was supported by the Howard Hughes Medical Institute (K.M.M.)and the Emmy Noether Program of the German Research Foundation (K.H.). 


\section{IntensivUpdate 2009}

\section{5. und 26. September 2009}

\section{Internistisches Intensiv-Update-Seminar}

$\mathrm{H}$ erzliche Einladung zum 1. IntensivUpdate in Wiesbaden!

Das IntensivUpdate ist eine neue Fortbildungsveranstaltung, die die sehr erfolgreichen internistischen UpdateSeminare ergänzt. Das IntensivUpdate wendet sich an Internisten und Internisten in Ausbildung, die in der internistischen Intensivmedizin dauerhaft oder im Rahmen der Rotation tätig sind.

In dem 2-tägigen Seminar wird Ihnen das renommierte Referententeam jedes Jahr den aktuellen internationalen Stand des Wissens in der internistischen Intensivmedizin präsentieren.

Besonderes Augenmerk gilt der Praxisrelevanz: In 14 interaktiven Teilseminaren referieren und kommentieren ausgewiesene Experten die wichtigsten Ergebnisse klinischer Studien des jeweils letzten Jahres. Dabei geht es vor allem um die abzuleitenden Konsequenzen für das ärztliche Handeln in der Intensivmedizin. Das Konzept unterscheidet sich damit deutlich von anderen Formaten und

Programm IntensivUpdate 2009

Freitag, 25. September 2009

09:45 Begrüßung

10:00 ACS und interventionelle Techniken Gerd Peter Meyer, Hannover

11:00 Kardiogener Schock und Cardiac-Assist-Systeme Karl Werdan, Halle/Saale

12:00 Speaker's Corner \& Pause

12:30 Rhythmusstörungen/ Endokarditis

Thorsten Lewalter, Paderborn

13:30 Enterale und parenterale Ernährung Konstantin Mayer, Gießen

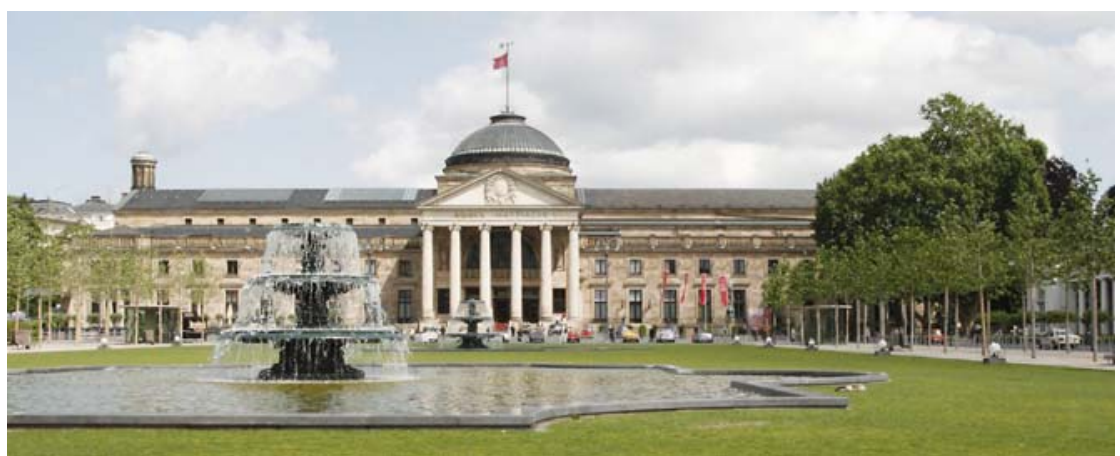

ist ein echtes Update des aktuellen Wissens.

Während der Vorträge und im Rahmen der Speaker's Corners besteht ausreichend Zeit, mit den Referenten $\mathrm{zu}$ diskutieren und sich unter Kollegen auszutauschen. Das Handbuch Internistische Intensivmedizin 2009 mit allen Manuskripten der Teilseminare ermöglicht Ihnen auch ein späteres Nachschlagen zu Hause.

Die Unabhängigkeit von Programmplanung und -inhalten ist zen- traler Bestandteil des Update-Konzeptes. Unser Dank gilt den Partnern des IntensivUpdates, der Novartis Pharma GmbH und der Karl Storz $\mathrm{GmbH} \&$ Co. KG, die das Konzept der unbeeinflussten Wissensweitergabe uneingeschränkt unterstützen.

Kommen Sie zum ersten IntensivUpdate nach Wiesbaden - es lohnt sich!

Prof. Dr. Tobias Welte, Hannover Prof. Dr. Karl Werdan, Halle/Saale Prof. Dr. Christian Ell, Wiesbaden
14:30 Speaker's Corner \& Mittagspause

15:30 Akutes Nierenversagen Dialyse

Danilo Fliser, Homburg/Saar

16:30 Speaker's Corner \& Pause

17:00 ARDS - Neue Beatmungsverfahren

Marius M. Hoeper, Hannover

18:00 Akute obstruktive Krise Nichtinvasive Beatmung, Weaning-Langzeitbeatmung Michael Pfeifer, Donaustauf

Samstag, 26. September 2009

08:00 Infektiologie: Erregerepidemiologie, Resistenzentwicklung Petra Gastmeier, Berlin
09:00 Management - Antibiotikatherapie

Tobias Welte, Hannover

10:00 Speaker's Corner \& Pause

10:30 Hepatologische Intensivmedizin Thomas Berg, Berlin

11:15 GI-Blutung, Pankreatitis, Darmischämie, Ileus Christian Ell/Jürgen Pohl, Wiesbaden

12:00 Speaker's Corner \& Mittagspause

12:45 Stroke - Enzephalitis/ Meningitis/CIP Stefan Schwab, Erlangen

13:45 Speaker's Corner \& Pause 
14:15 Sepsis

Frank M. Brunkhorst, Jena

15:15 Hot Topic: Hypothermie Michael Joannidis, Innsbruck

16:00 Speaker's Corner \& Ende der Veranstaltung

Informationen zum IntensivUpdate 2009

Wissenschaftliche Leitung

Prof. Dr. Tobias Welte, Hannover Prof. Dr. Karl Werdan, Halle/Saale Prof. Dr. Christian Ell, Wiesbaden

Seminarort

Kurhaus-Kolonnaden Wiesbaden, Kurhausplatz 1, 65189 Wiesbaden
Anmeldung

Im Internet unter: www.intensivupdate.com

Per Fax unter: +49 (611) 204809-10

Teilnahmegebühr

$300,-€$ bei Voranmeldung und Bezahlung bis zum 18. September 2009

$350,-€$ bei Registrierung und Bezahlung vor Ort

$250,-€$ vorab bzw. 300,- $€$ vor Ort für Assistenzärzte in Ausbildung (Nachweis erforderlich). Alle Preise inkl. 19\% MwSt.

Die Teilnahmegebühr beinhaltet

- Seminarteilnahme am 25. und 26. September 2009

- Handbuch Internistische Intensivmedizin 2009
- CD-ROM aller Vortragspräsentationen (Auslieferung voraussichtlich November 2009)

- Pausenverpflegung an beiden Tagen

Organisation und Teilnehmerregistrierung

wikonect $\mathrm{GmbH}$, Wiesbaden Telefon: +49 (611) 204809-0

Konzeptentwicklung und Veranstalter med update GmbH, Wiesbaden www.med-update.com 


\section{Mitteilungen der Gesellschaft für Innere Medizin Sachsen-Anhalt}

Vorsitzender

Prof. Dr. M. Plauth, Klinik für Innere Medizin, Städtisches Klinikum Dessau, Auenweg 38, 06847 Dessau-Roßlau

\section{Redaktion}

M. Plauth, Dessau-Roßlau,

(Vorsitzender)

\section{Jahrestagung der Gesellschaft}

\section{für Innere Medizin Sachsen-Anhalt e.V.}

\section{3. und 14. November 2009 in Dessau-Roßlau}

iebe Kolleginnen und Kollegen,
sehr geehrte Damen und Herren, ich möchte Sie herzlich zur Jahrestagung der Gesellschaft für Innere Medizin Sachsen-Anhalt am 13. und 14. November 2009 in Dessau-Roßlau einladen. Wir können uns auf ein anregendes Tagungsprogramm freuen, das mit seinen Referaten und freien Beiträgen das gesamte Spektrum der Inneren Medizin überspannen wird. In bewährter Weise wird sich am Freitagvormittag das Pflegeseminar in bekannt interdisziplinärer Weise mit aktuellen Themen wie dem neuen Standard zum Ernährungsmanagement befassen. Für die Kolleginnen und Kollegen in der Facharztweiterbildung besteht am Freitagvormittag die Gelegenheit, in Refresherkursen den Kenntnisstand in der Befundung von Röntgenbildern in der Notfallversorgung, der Labordiagnostik

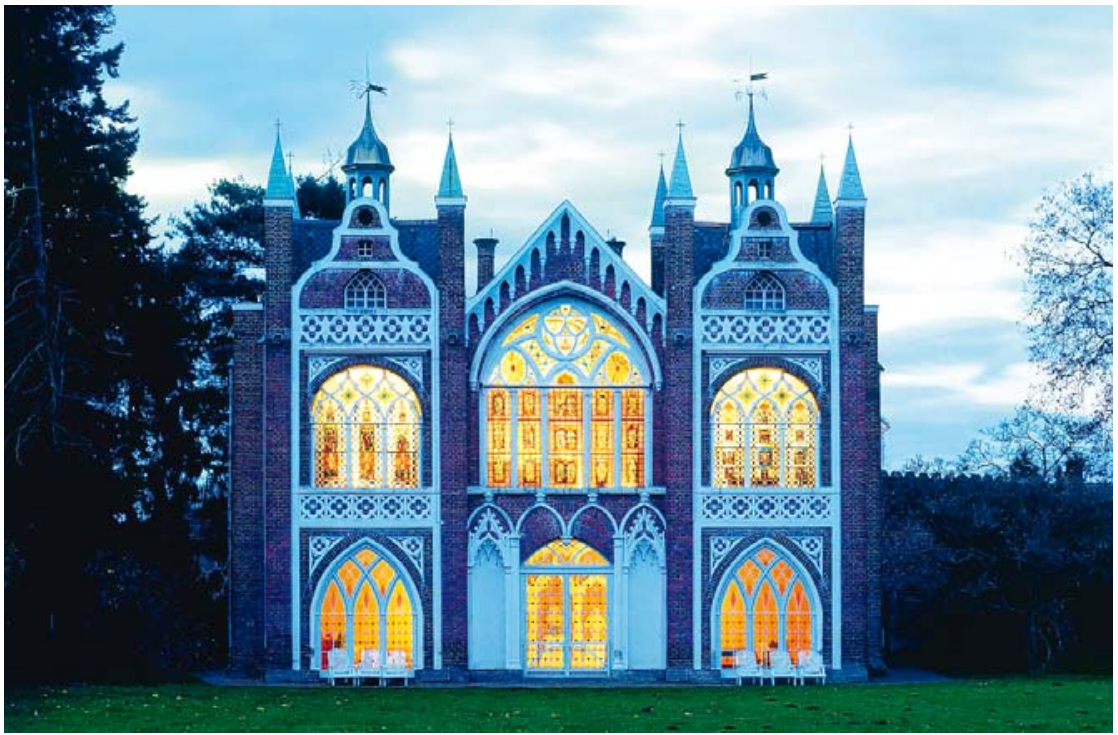

Gotisches Haus in Wörlitz. und der kardiopulmonalen Reanimation zu aktualisieren. Im Hauptprogramm werden neben neuen diagnostischen und therapeutischen Strategien auch Fragen zum rationalen Vorgehen bei in der Praxis häufigen und bedeutsamen „Alltagsproblemen“ von der Synkope bis zur Reisediarrhö angesprochen. Die Tagung soll uns Gelegenheit bieten, den Kontakt aller in der Region internistisch tätigen Kolleginnen und Kollegen nicht nur in den virtuellen Medien, sondern auch im persönlichen Gespräch zu pflegen. Dabei kommt den freien Beiträgen eine ganz besondere Bedeutung zu, denn sie bilden stets eine hervorragende $\mathrm{Ba}-$ sis, um entweder in der Diskussion am Poster oder in der bewährten Sitzung der kommentierten Referate am Samstag diesen kollegialen Dialog zu vertiefen. Die Gesellschaft widmet den

freien Beiträgen ganz bewusst einen großen Raum, um allen internistischen Kollegen die Möglichkeit zu geben, ,unsere“ Jahrestagung aktiv mitzugestalten und Akzente zu setzen.

Wir freuen uns sehr auf Ihre Teilnahme an der Jahrestagung unserer Gesellschaft und darauf, Sie in DessauRoßlau begrüßen zu können.

Prof. Dr. Mathias Plauth,

Vorsitzender der Jahrestagung 2009

Wissenschaftliche Leitung

Prof. Dr. M. Plauth

Klinik für Innere Medizin

Städtisches Klinikum Dessau

Auenweg 38, 06847 Dessau-Roßlau

Tagungsort

Steigenberger Hotel Fürst Leopold Friedensplatz, 06846 Dessau-Roßlau www.dessau.steigenberger.de

\section{Information}

Silke Serfling/Heike Nemczak Sekretariat Klinik für Innere Medizin Städtisches Klinikum Dessau Auenweg 38, 06847 Dessau-Roßlau Telefon 0340/501 1275

Fax 0340/501 1210

Anmeldung der freien Beiträge

Bis zum 15. 9. 2009 über

www.imsa.de

Pflege-Seminar

13. 11. 2009, Anmeldung über www.imsa.de

Mitgliedschaft

Werden Sie Mitglied. Die Mitgliedschaft ist beitragsfrei. Mitgliedsantrag über www.imsa.de 
Sonstige Veranstaltungen

Kardio Intermezzo 2009
Bad Kissingen
18.-19.09.2009
Schirmherrschaft DGIM
$\begin{array}{ll}\text { Wissensch. } & \text { Prof. Dr. med. Sebastian Kerber } \\ \text { Organisation } & \text { Prof. Dr. med. Burghard } \\ & \text { Schumacher } \\ \text { Prof. Dr. med. Anno Diegeler }\end{array}$
$\begin{array}{ll}\text { Veranstalter: } & \text { Herz- und Gefäß-Klinik } \\ \text { Bad Neustadt }\end{array}$
$\begin{array}{ll}\text { Hauptthemen: } & \text { Kardiologie } \\ \text { Info und } & \text { www.kardio-intermezzo.de } \\ \text { Anmeldung: } & \text { www.kelcon.de/termine.html }\end{array}$

\section{Jahreskongress der Deutschen Gesellschaft für Rheumatologie (DGRh) Köln}

23.-26.09.2009

DG Schirmherrschaft DGIM

Tagungs-

präsident:

Veranstalter:

Hauptthemen:

Info und

Anmeldung:
PD Dr. Andrea Rubbert-Roth Prof. Dr. Gerd Horneff

Deutsche Gesellschaft für Rheumatologie

on der Pathogenese zur Klinik Interdisziplinäre Rheumatologie Kinder- und Jugendrheumatologie Rheumatoide Arthritis Vaskulitis und Kollagenosen Genetik in der Rheumatologie Versorgung in der Rheumatologie Rheumaorthopädie

www.dgrh.de

\section{Kongress der Südwest- deutschen Gesellschaft für Innere Medizin \\ Ravensburg}

25.-26.09.2009

Schirmherrschaft DGIM

Tagungspräsident:

Veranstalter: Südwestdeutsche Gesellschaft für

Hauptthemen: Internisten in Bewegung“

Info und www.medcongress.de Anmeldung: Innere Medizin

Prof. Dr. G. J. Wiedemann 


\section{Update-Seminare - Fortbildung für Ärzte auf höchstem Niveau}
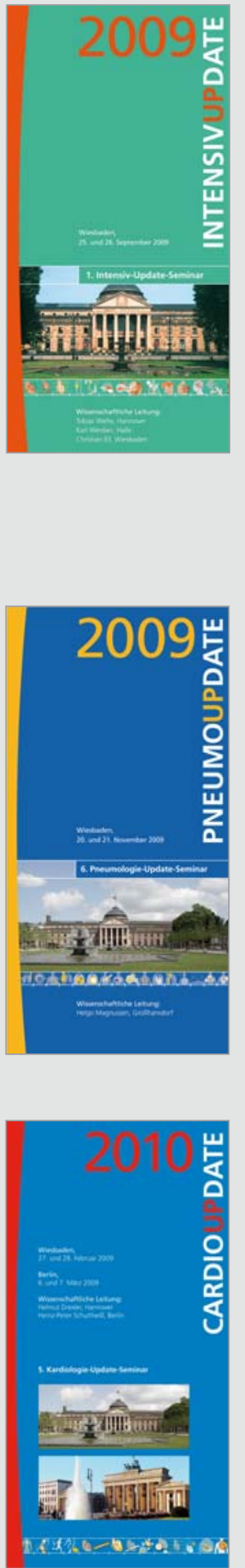

\section{INTENSIVUPDATE2009}

25./26. September 2009

1. Internistisches IntensivUpdate-Seminar

Veranstaltungsort:

Wiesbaden, Kurhaus Kolonnaden Informationen und Anmeldung: www.intensiv-update.com

Wissenschaftliche Leitung:

Prof. Dr. Tobias Welte

Prof. Dr. Karl Werdan

Prof. Dr. Christian Ell

Schirmherrschaft:

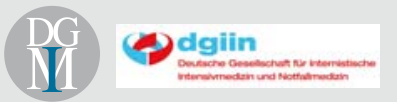

PNEUMOUPDATE2009

20. / 21. November 2009

6. Pneumologie-Update-Seminar Veranstaltungsort:

Wiesbaden, Kurhaus

Informationen und Anmeldung: www.pneumo-update.com

Wissenschaftliche Leitung:

Prof. Dr. Helgo Magnussen

Schirmherrschaft:

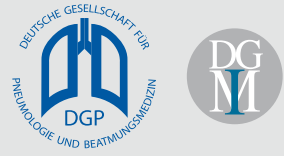

\section{CARDIOUPDATE2010}

Wiesbaden 26./27. Februar 2010 Berlin 05./ 06. März 2010

5. Kardiologie-Update-Seminar Veranstaltungsorte:

Wiesbaden, Kurhaus

Berlin, bcc

Informationen und Anmeldung: www.cardio-update.com

\section{Wissenschaftliche Leitung:}

Prof. Dr. Helmut Drexler

Prof. Dr. Heinz-Peter Schultheiß

Schirmherrschaft:

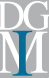

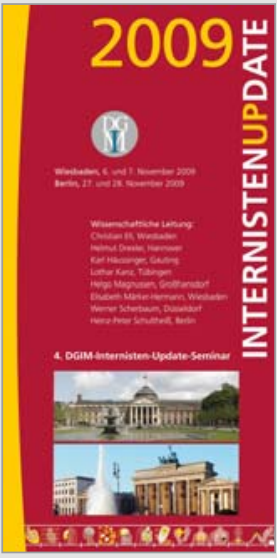

Schirmherrschaft:
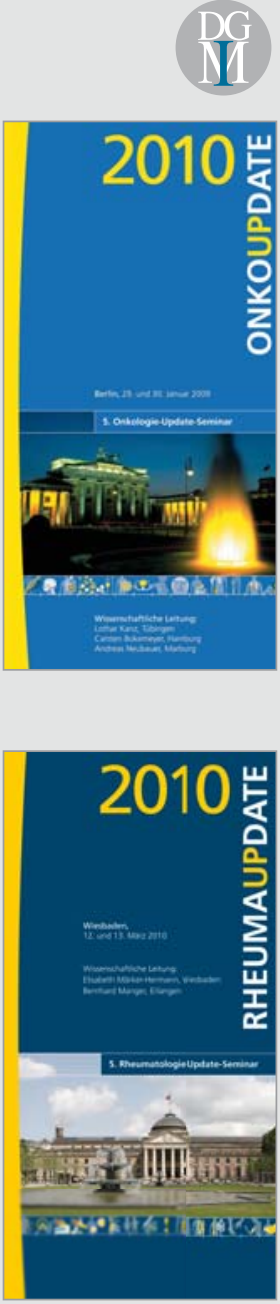

INTERNISTEN UPDATE 2009

Wiesbaden 06./ 07. November 2009 Berlin 27. / 28. November 2009

4. DGIM-Internisten-Update-Seminar Veranstaltungsorte:

Wiesbaden, Kurhaus Berlin, bcc

Informationen und Anmeldung: www.internisten-update.com

Wissenschaftliche Leitung:

Prof. Dr. Christian Ell

Prof. Dr. Helmut Drexler

Prof. Dr. Karl Häussinger

Prof. Dr. Lothar Kanz

Prof. Dr. Helgo Magnussen

Prof. Dr. Elisabeth Märker-Hermann

Prof. Dr. Werner Scherbaum

Prof. Dr. Heinz-Peter Schultheiß

\section{ONKO DATE}

29. / 30. Januar 2010

5. Onkologie-Update-Seminar

Veranstaltungsort:

Berlin, bcc

Informationen und Anmeldung:

www.onko-update.com

Wissenschaftliche Leitung:

Prof. Dr. Lothar Kanz

Prof. Dr. Carsten Bokemeyer

Prof. Dr. Andreas Neubauer

Schirmherrschaft:

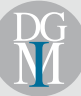

RHEUMA UPATE 2010

Wiesbaden 12./ 13. März 2010

5. Rheumatologie-Update-Seminar

Veranstaltungsort:

Wiesbaden, Kurhaus

Informationen und Anmeldung:

www.rheuma-update.com

Wissenschaftliche Leitung:

Prof. Dr. Elisabeth Märker-Hermann

Prof. Dr. Bernhard Manger

Schirmherrschaft:

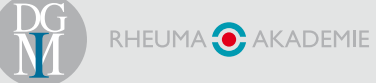

med

date
Konzeptentwicklung und Veranstalter:

med update $\mathrm{GmbH}, 65203$ Wiesbaden, Hagenauer Straße 53

www.med-update.com

E-Mail: info@med-update.com
Tel.: +49 $61173658-0$

Fax: +49 $61173658-10$ 


\section{Intensivkurse Innere Medizin - Vorbereitung auf die Facharztprüfung und Refreshing 2009}

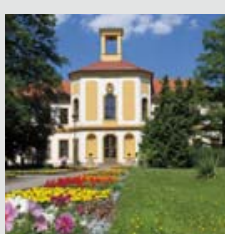

Dresden
Leitung

Anmeldung/ Organisation
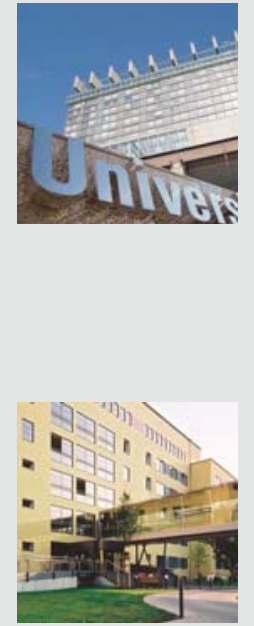

Erfurt

Leitung

Anmeldung/

Organisation

Veranstaltungsor

07.09.-11.09.2009

II Schirmherrschaft DGIM

Dr. med.Wollschläger

Prof. Dr. med. Schellong

Städt. Klinikum Dresden-Friedrichstadt

III. Medizinische Klinik

Frau Gertraude Liebold

Friedrichstraße 41

01067 Dresden

Telefon (+49/3519) 48011-30

Fax -49

Liebold-ge@khdf.de

Veranstaltungsort Städt.Klinikum Dresden-Friedrichstadt Friedrichstraße 41

01067 Dresden

Köln/Bonn 07.09.-12.09.2009

II Schirmherrschaft DGIM

Dr. P. Walger (Bonn)

PD Dr.J. Müller-Ehmsen (Köln)

Frau Ch.Wihnalek (Sekretariat)

Telefon (+49/228) 287-22502

Fax -22634

christel.wihnalek@gmx.de walger@uni-bonn.de

Universität Bonn

Anatomisches Institut, Hörsaal B

Nussallee 10

53115 Bonn

14.09.-18.09.2009

III Schirmherrschaft DGIM

Prof.Dr. med. U. Schmidt

Dr.med.K.-M.Deppermann

PD Dr.med. H. Lapp

Prof.Dr.med. M. Herold

Anmeldung

Akademie für ärztliche Fort- und

Weiterbildung der LÄK Thüringen

Frau Schneider

Postfach 100740,07707 Jena

Telefon (+49/3641) 6141-43

Fax -49

schneider.akademie@laek-thueringen.de HELIOS Klinikum Erfurt, Zentrum für Innere Medizin, 2. Medizinische Klinik

Frau Iris Effenberger

Nordhäuser Straße 74

99089 Erfurt

Telefon (+49/361) 7812-471, Fax -472 iris.effenberger@helios-kliniken.de www.helios-kliniken.de

Veranstaltungsort Helios Klinikum Erfurt, Auditorium

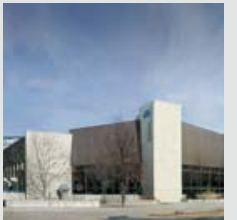
München -
Klinikum
rechts der Isar
Leitung
Anmeldung/
Organisation
14.09.-18.09.2009
Schirmherrschaft DGIM
PD Dr.med. Bruno Neu, München
Klinikum rechts der Isar
II. Med. Klinik
Frau Anna Heering
Telefon $(+49 / 89)$ 4140-4386
Fax -4115
anna.heering@lrz.tum.de
www.med2.med.tu-muenchen.de
Veranstaltungsort Klinikum rechts der Isar, Hörsaal D
Ismaninger Straße 22
81675 München

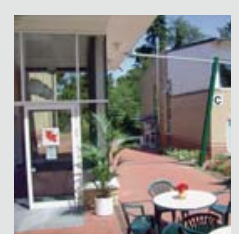

Bad Segeberg
Leitung
Anmeldung/
Organisation

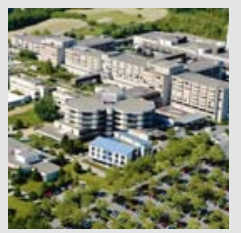

Wiesbaden

Leitung

Anmeldung/

Organisation

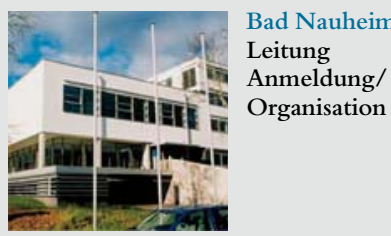

Veranstaltungsort Hörsaal des Dr. Peter-Jäger-

Ausbildungszentrums

(neben Dr. Horst-Schmidt-Kliniken)

Ludwig-Erhard-Straße 98, 65199 Wiesbaden

23.11.-28.11.2009

Prof. Dr. med.W. Fassbinder, Fulda

Akademie für ärztliche Fort- und

Weiterbildung

der Landesärztekammer Hessen

Frau Adelheid Zinkl

Carl-Oelemann-Weg 5-7

61231 Bad Nauheim

Telefon $(+49 / 6032) 782-227$

Fax -229

adelheid.zinkl@laekh.de

www.laekh.de

Veranstaltungsort Fortbildungszentrum der LÄK Hessen

Carl-Oelemann-Weg 5-7

61231 Bad Nauheim

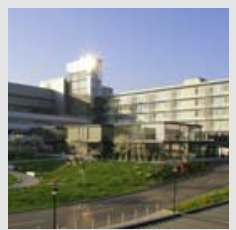

Stuttgart

Leitung

Anmeldung/

Organisation

$\begin{array}{ll} & \text { Auerbachstraße 110 } \\ & \text { 70376 Stuttgart } \\ & \text { Telefon (+49/711) 8101-3406 } \\ & \text { Fax -3793 } \\ & \text { zim1@rbk.de } \\ \text { Veranstaltungsort } & \text { Hörsaal des Robert-Bosch-Krankenhauses } \\ & \text { Stuttgart } \\ & \text { Auerbachstraße110 } \\ & \text { 70376 Stuttgart }\end{array}$

Weitere Informationen unter:

http://www.dgim.de/termine/termine_intensivkurse.html

Verantwortlich: Prof. Dr. Hans-Peter Schuster, Generalsekretär,

Deutsche Gesellschaft für Innere Medizin e.V., Irenenstraße 1, 65189 Wiesbaden

Telefon (+49/611) 2058040-0, Fax (+49/611) 2058040-46, E-Mail: info@dgim.de, Internet: www.dgim.de 